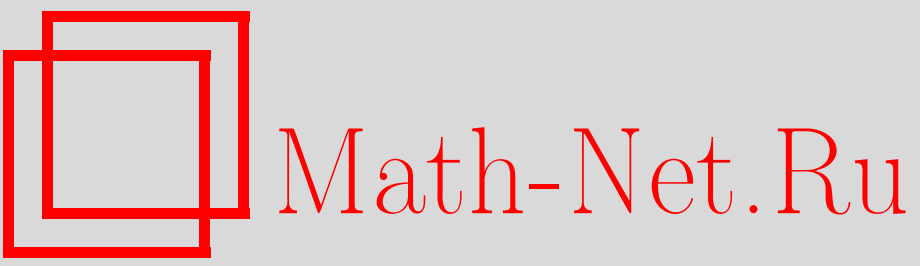

Е. М. Крейнес, Паразитические решения систем уравнений на функции Белого в пространствах Гурвица, УМН, 2001, том 56, выпуск 6, 155-156

DOI: https://doi.org/10.4213/rm464

Использование Общероссийского математического портала Math-Net.Ru подразумевает, что вы прочитали и согласны с пользовательским соглашением

http://www.mathnet.ru/rus/agreement

Параметры загрузки:

IP : 54.205 .225 .156

26 апреля 2023 г., 13:22:24 


\title{
ПАРАЗИТИЧЕСКИЕ РЕШЕНИЯ СИСТЕМ УРАВНЕНИЙ НА ФУНКЦИИ БЕЛОГО В ПРОСТРАНСТВАХ ГУРВИЦА
}

\author{
Е. М. КРЕЙНЕС
}

Поиск различных конструктивных подходов к визуализации эквивалентности категории детских рисунков (вложенные графы на поверхностях) и категории пар Белого (алгебраическая кривая $X$ и непостоянная рациональная функция $\beta$ на ней, имеющая не более трех различных критических значений), доказанной в [1], начинается с работы А. Гротендика [2] и является актуальной задачей современной математики, см. [3]-[8].

В настоящей работе рассматривается проективная прямая $X=\mathbf{P}^{1}$. В этом случае соответствующие детские рисунки оказьваются плоскими граффами, а критическими значениями функции Белого можно без ограничения общности считать 0,1 и $\infty$. При этом мы можем использовать глобальные координаты на $\mathbf{P}^{1}$ и задать в явном виде систему уравнений на функцию Белого, отвечающую данному рисунку.

Пусть $\Gamma$ - некоторьй связньй плоский граф, $A_{1}, \ldots, A_{k} \in \mathbf{P}^{1}-$ координаты его вершин, $C_{1}, \ldots, C_{m} \in \mathbf{P}^{1}$ - координаты "центров" его граней, $\left\langle\alpha_{1}, \ldots, \alpha_{k} \mid \gamma_{1}, \ldots, \gamma_{m}\right\rangle$ - набор 0-валентностей и 2 -валентностей граффа Г. Легко видеть, что функция Белого этого графа имеет вид $\beta(z)=c \frac{\left(z-C_{1}\right)^{\gamma_{1}} \cdots\left(z-C_{m}\right)^{\gamma_{m}}}{\left(z-A_{1}\right)^{\alpha_{1}} \cdots\left(z-A_{k}\right)^{\alpha_{k}}}$ для некоторого конечного ненулевого элемента $c$ (в случае если какая-то из величин $A_{i}, i=1, \ldots, k$, или $C_{j}, j=1, \ldots, m$, равняется бесконечности, мы опускаем соответствующий множитель). Заметим, что $\beta$ является элементом пространства Гурвица $\mathscr{H}_{0,2 n}$ рода 0 степени $2 n$, где $n$ - количество ребер графо $\Gamma$.

Переходя к однородным координатам, замечая, что функция $\beta$ не зависит от порядка сомножителей, и используя после этого в отдельности для числителя и для знаменателя функции $\beta$ тот факт, что $\frac{\left(\mathbf{P}^{1}\right)^{d}}{S_{d}} \cong \mathbf{P}^{d}$ для любого натурального $d$, получим вложение $\tau: \mathscr{H}_{0,2 n} \rightarrow \mathbf{P}^{1} \times$ $\mathbf{P}^{2 n} \times \mathbf{P}^{2 n}$. Тем самым, функцию $\beta$ можно рассматривать как элемент компактного (в естественной топологии) пространства $\mathbf{P}^{1} \times \mathbf{P}^{2 n} \times \mathbf{P}^{2 n}$. В этом пространстве для каждого набора 0 - и 2-валентностей $\left\langle\alpha_{1}, \ldots, \alpha_{k} \mid \gamma_{1}, \ldots, \gamma_{m}\right\rangle$ нами задается система уравнений, определяющая функцию $\beta$ и назьваемая системой Гротендика-Белого. Эта система является покомпонентной записью утверждения о том, что числитель функции $(1-\beta)$ является полньпм квадратом. Для каждого решения $\left(c ; A_{1}, \ldots, A_{k} ; C_{1}, \ldots, C_{m}\right)$ системы Гротендика-Белого рассмотрим $n c e в д о$ функцию Белого: $B=c \frac{\left(z-C_{1}\right)^{\gamma_{1}} \cdots\left(z-C_{m}\right)^{\gamma_{m}}}{\left(z-A_{1}\right)^{\alpha_{1}} \cdots\left(z-A_{k}\right)^{\alpha_{k}}}$.

Решение $\left(c ; A_{1}, \ldots, A_{k} ; C_{1}, \ldots, C_{m}\right)$ называется паразитическим, если соответствующая псевдофункция Белого $B$ является функцией Белого некоторого графа $\bar{\Gamma}$, набор 0 - и 2 -валентностей которого отличается от $\left\langle\alpha_{1}, \ldots, \alpha_{k} \mid \gamma_{1}, \ldots, \gamma_{m}\right\rangle$, или если $B$ вообще не является функцией Белого. Будем говорить, что заданный набор валентностей (или графф Г с заданным набором валентностей) обладает геометрическим и негеометрическим паразитическими решениями в первом и втором случаях соответственно.

Теорема 1. Пусть граф Г обладает геометрическим паразитическим решением. Тогда это решение лежит на границе множества $\tau\left(\mathscr{H}_{0,2 n}\right)$ в пространстве $\mathbf{P}^{1} \times$ $\mathbf{P}^{2 n} \times \mathbf{P}^{2 n}$

Теорема 2. Граф обладает или не обладает паразитическим решением одновременно со своим двойственным графом. Паразитическое решение для графа является геометрическим тогда и только тогда, когда является геометрическим паразитическое решение для двойственного графа.

Работа выполнена при частичной поддержке Российского фонда фундаментальных исследований (гранты № 00-15-96128 и № 99-01-00382). 
Теорема 3. Пусть $\Gamma_{\lambda \mu}-$ плоский граф следующего вида:

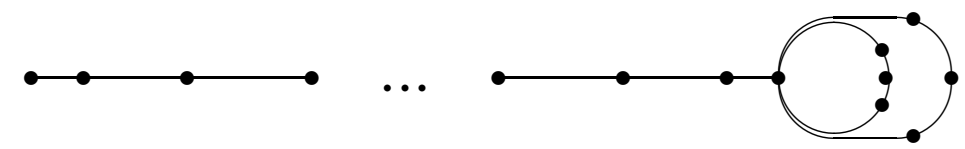

состоящий из "ручки", на которой расположено $\lambda$ точек валентности 2 и двух петель, обладающих общей точкой, на каждой из которых расположено по $\mu$ точек валентности 2. Для всех $\lambda$ и $\mu$ граф $\Gamma_{\lambda \mu}$ обладает геометрическим паразитическим решением, соответствуюшим графу $\overline{\Gamma_{\lambda \mu}}$, который может быть получен из $\Gamma_{\lambda \mu}$ склеиванием петель друг с другом и имеет следующий вид:

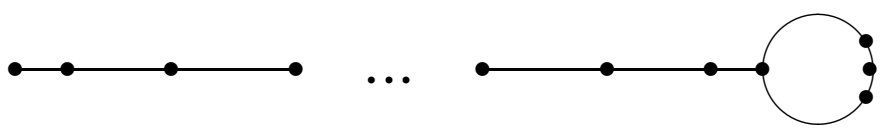

СледСтвиЕ 1. Существуют бесконечные семейства графов, обладающих геометрическими паразитическими решениями.

ПримеР. Граф $\Gamma_{0,2,0}$

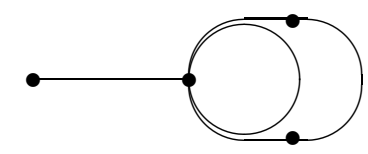

обладает однопараметрической системой паразитических решений, которые при всех значениях параметра, кроме двух, являются негеометрическими.

СледСтвиЕ 2. Существуют графы, обладающие бесконечным количеством негеометрических паразитических решений.

\section{СПИСОК ЛИТЕРАТУРЫ}

[1] G. B. Shabat, V. A. Voevodskij // Progr. Math. 1990. V. 88. P. 199-227. [2] A. Grothendieck // London Math. Soc. Lecture Notes Ser. 1997. V. 243. P. 3-43. [3] J. Bètrèma, D. Pérè, A. Zvonkine. Plane Trees and their Shabat Polynomials. Catalog // Rapports Internes de LaBRI №75-92, Bordeaux, 1992. [4] Н. М. Адрианов, Ю. Ю. Кочетков, Г. Б. Шабат, А. Д. Суворов // Фундамент. прикл. матем. 1995. Т. 1. № 2. С. 377-384. [5] Yu. Yu. Kochetkov // Proceedings of the 12-th International Conference FPSAC-00. Berlin: Springer-Verlag, 2000. P. 447-454. [6] G. Shabat // Proceedings of the 12-th International Conference FPSAC-00. Berlin: Springer-Verlag, 2000. P. 575-581. [7] E. M. Крейнес, Г. Б. Шабат // Фундамент. прикл. матем. 2000. Т. 6. №3. С. 289-292. [8] G. Shabat, A. Zvonkine // Contemp. Math. 1994. V. 178. P. 233-275. 